\title{
Methods to Eye Gaze Interaction for Authentication
}

\author{
Yvonne Hwei-Syn Kam, Kar Wing Kwong, Vik Tor Goh
}

\begin{abstract}
One of the methods touted to reduce shoulder surfing during onscreen authentication is eye gaze input. Eye gaze authentication methods have thus far relied on a few methods for selection of on screen objects, namely dwell time, blinking, eye gestures and pursuit of moving objects. This paper surveys the literature on the various approaches. From analysis of the advantages and drawbacks of the surveyed approaches, we find that the dwell method has advantages in security and usability. We then describe a method designed previously by the authors that enhances the dwell method by making use of peripheral vision. The proposed method is in the ideation stage.
\end{abstract}

Keywords - survey, shoulder surfing, gaze, eye tracking, peripheral vision.

\section{INTRODUCTION}

Eye gaze tracking is a promising way of interacting with the computer which is faster than a mouse [1]. It has been used for security applications for password entry where it is touted to be more shoulder surfing resistant than conventional graphical passwords [2] because selection is done with the eyes which is not as easily observable by attackers. The four usual ways of gaze based interaction are categorized according to what kind of eye movements are required. They are pursuit based, dwell based, blink based and gesture based. This paper is arranged as follows: Firstly, a survey of related works under each category of gaze based interaction is presented. Next, we explain the working of a dwell based method which uses peripheral vision developed by the authors. This is followed by a discussion on the merits and demerits of this proposed method and lastly followed by the conclusion.

\section{RELATED WORK}

We begin by looking at the various different gaze interaction techniques.

\section{A. Pursuit Based}

Pursuit is following the target with your eyes. This kind of interaction is used by two input systems for text, Dasher [3] and Stargazer [4]. It is also used by Pursuits [5] where it is used for object selection for interaction. It was also used by Cymek et al. [6] for selecting objects in their method. This

Revised Manuscript Received on August 16, 2019.

Yvonne Hwei-Syn Kam, Faculty of Engineering, Multimedia University, Cyberjaya, Malaysia.

Kar Wing Kwong, Faculty of Engineering, Multimedia University, Cyberjaya, Selangor.

Vik-Tor Goh, Faculty of Engineering, Multimedia University, Cyberjaya, Malaysia. method is good because it does not require calibration. However, it is possibly more easily shoulder surfed because the movement of the eyes follows the trajectory of only one of the moving objects. Also, the password space is somewhat small because of limited pursuit paths thus limiting the number of objects. Paths must be dissimilar enough to be distinguishable by the algorithm or the objects must move at different enough speeds [5]. Also, the interface has to be dynamic, meaning that there are moving objects. Thus it is not suitable for static interface elements or text because text needs to be stationary to be read. It is more difficult to read text which is moving, especially long text. If pursuit is performed at length, the perpetual movement could cause fatigue or confusion [5]. Another problem is vulnerability to head movements. If the user moves his head to pursue an object, making a large movement, then his eye trajectory will not be the same as the object pursued.

\section{B. Dwell Based}

Dwell based interaction works by dwelling on an object with your eyes for a certain amount of time for the purpose of object selection. Examples of methods that use dwell based approach are [7], [8], [9]. Dwell times for activation of a selection are usually between $100 \mathrm{~ms}$ to $1000 \mathrm{~ms}$ [10]. Shorter dwell times make for faster interaction but are more prone to errors, whereas longer dwell times are more strenuous for the user's eyes and time consuming but reduce errors [11]. Another study [12] says that the participants regarded the dwell based method as more intuitive and easier to get used to as well as less tiring than a blink based method. However, dwell based interaction could suffer from the Midas Touch problem [13]. Midas Touch is when everything the user fixates at, whether deliberate or otherwise is interpreted as a selection. This is a problem because fixations occur even when a user is interested to look a little longer at an object, but not necessarily wants to select it. The solution is to set a threshold time that is sufficient yet not too long that the user is tired. Dwell based methods also need eye calibration [17].

Dwell based methods are generally more secure against shoulder surfing. This is because it is easier for an attacker to identify an eye gesture or pursuit path by comparing the eye movement to what is shown on screen than to identify the exact target that the user is fixated at, especially if there are many targets which are somewhat close together.

\section{Blink Based}

Blink based is where objects are selected by blinking while looking at them. A blink is a prolonged absence of gaze detection. The last fixation point before the blink is taken as the selected object. Examples of blink based

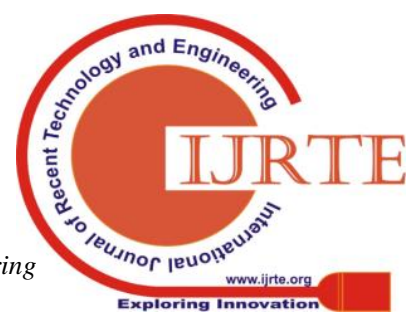


systems are [14] and [15]. Blink based methods have less errors and are more accurate than dwell based methods as shown by a study[12]. However, participants of the study rated it as less intuitive and more tiring than a dwell based method [12].

TABLE I. SUMMARY OF METHODS

\begin{tabular}{|c|c|c|c|}
\hline Approach & Examples & Advantages & Drawbacks \\
\hline Pursuit & $\begin{array}{l}{[3],[4],[5],} \\
{[6]}\end{array}$ & - Does not require calibration & $\begin{array}{l}\text { - Can be shoulder surfed because the movement of } \\
\text { the eyes follows the trajectory of only one of the } \\
\text { moving objects. } \\
\text { - Password space is somewhat small because of } \\
\text { limited pursuit paths thus limiting the number of } \\
\text { objects. } \\
\text { - Interface has to be dynamic. Not suitable for } \\
\text { static interface elements or text } \\
\text { - Constant movement might be a source of } \\
\text { confusion or fatigue for users if used for longer } \\
\text { periods of time [5] } \\
\text { - Vulnerability to head movements }\end{array}$ \\
\hline Dwell & {$[7],[8],[9]$} & $\begin{array}{l}\text { - More secure against shoulder surfing } \\
\text { than pursuit /gesture approaches } \\
\text { - Implementing higher resolution } \\
\text { images and PINs will provide more } \\
\text { authentication combination [8] } \\
\text { - More intuitive and easier to get used } \\
\text { to as well as less tiring than a blink } \\
\text { based method. [12] }\end{array}$ & $\begin{array}{l}\text { - Shorter dwell times are more prone to errors, } \\
\text { longer dwell times could be strenuous [11] } \\
\text { - May have Midas Touch problem [13] } \\
\text { - Needs eye calibration [17] }\end{array}$ \\
\hline Blink & {$[14],[15]$} & $\begin{array}{l}\text { - More accurate, less errors than dwell } \\
\text { approach [12] }\end{array}$ & $\begin{array}{l}\text { - Participants rated it as less intuitive, and more } \\
\text { tiring than dwell based [12] }\end{array}$ \\
\hline Gesture & $\begin{array}{l}{[17],[18],} \\
{[20]}\end{array}$ & $\begin{array}{l}\text { - No need to have calibration } \\
\text { performed } \\
\text { - Single stroke gesture systems [20] are } \\
\text { faster than dwell time or blink based } \\
\text { interactions }\end{array}$ & $\begin{array}{l}\text { - More susceptible to shoulder surfing because the } \\
\text { same eye gesture can be copied by an attacker } \\
\text { - Smaller password space: only a few single stroke } \\
\text { gestures available }\end{array}$ \\
\hline
\end{tabular}




\section{Gesture Based}

Gesture based is when eye gestures are used to perform object selection or drawing. They can be performed by having certain eye gestures act as symbols to indicate certain operations [16]. Other methods use eye gestures to draw letters and numbers [17]. Gaze operations often match eye movements to an on screen template, but in more recent works, the gestures are trained beforehand and performed from memory [18]. Studies [19] have shown that this takes less time compared to using templates while being just as reliable. Eye gestures are performed as a series of strokes or as a single stroke. Single stroke gesture systems [20] are faster than dwell time or blink based interactions. However there are only a few single stroke gestures, thus limiting the number of gestures that can be performed, thus reducing the password space. Eye gestures are, in general, more susceptible to shoulder surfing because the same eye gesture can be copied by an attacker. There is no need to have calibration performed, so it is not tied to the personal eye profile of a person.

A summary of the eye gaze interaction approaches is shown in Table I.

\section{DWELL METHOD UTILISING PERIPHERAL VISION}

Dwell approaches can be used to seamlessly replace mouse based interaction in existing interfaces. It also has higher resistance to shoulder surfing and larger password space than pursuit or gesture approaches. It is also intuitive, easier and less tiring than blink based methods. The approach is able to benefit from added enhancement from peripheral vision such as motion detection.

\section{A. Peripheral Vision}

Peripheral vision is detected in the high acuity area of the vision field of humans, called the fovea. This field of view is about 2 degrees in diameter. This angle is roughly equivalent to viewing a thumbnail from a distance of an arm's length [21]. Outside the fovea is the peripheral area. It is sensitive to light, shade and motion.

The peripheral area gives the user information on changes in the scene or nearby movement. Sudden movements in the peripheral area can quickly attract the user's attention [22]. Our system was designed to take advantage of these eye characteristics in that it uses the small area that the user can focus on and changes the colour of the object within the user's focus and also has movement in the periphery to signal to the user the next change of colour.

Peripheral vision is used in computer games where precise gameplay, fast motion and shooting are involved. The key idea of the approach is to use peripheral vision to spot changes in the surrounding area around a target and respond to the changes. User's reaction is based on what is seen from his peripheral vision. For example in a game like Pacman, when a player sees a ghost coming from a distance starting from the corner of his eye, he will move Pacman to another direction to avoid the oncoming ghost or go towards the ghost (in the case that Pacman has swallowed the Power Pellets!).

The approach to take advantage of peripheral vision is made more effective by involving flicker and motion to attract gaze. We now describe a scheme that incorporates both dwell time and change detection in nearby objects by using peripheral vision. A brief description of the method was given in a short paper presentation [23]. In this paper it is described in detail.

\section{B. Layout}

Fig. 1 shows an example of an image on which to overlay the objects to be fixated on. An image is used as a reference to help users remember the positions of their password target Fig. 2 shows the layout of the interface. It has crosshairs (cross-shaped targets) indicating the targets that the user can choose from

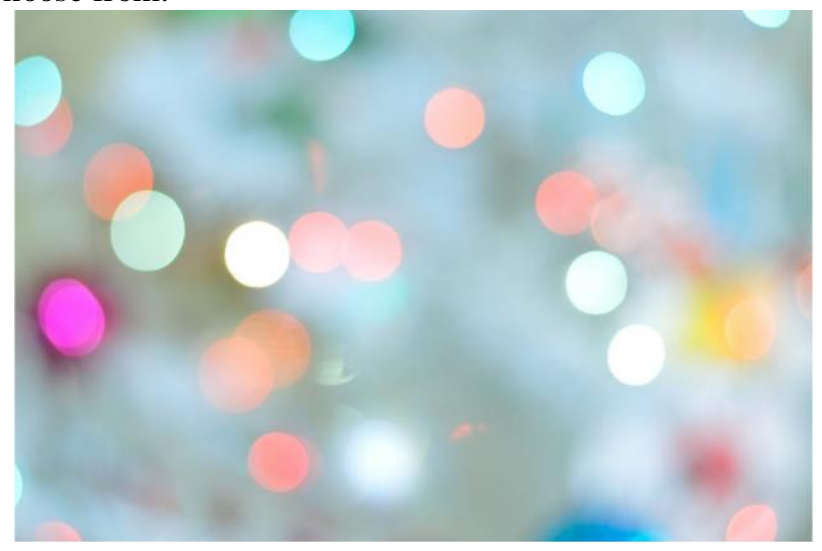

Figure 1. Example of background image

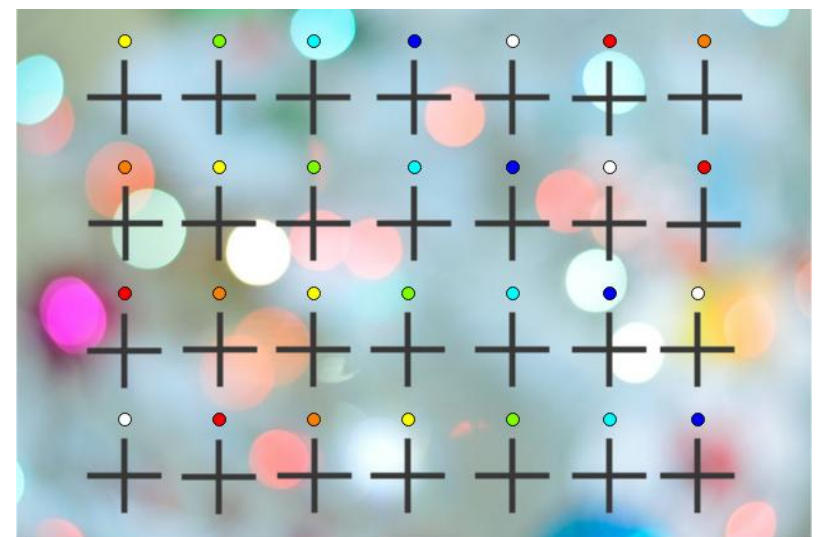

Figure 2. Layout of the user interface with changing circle colours (cyclic)

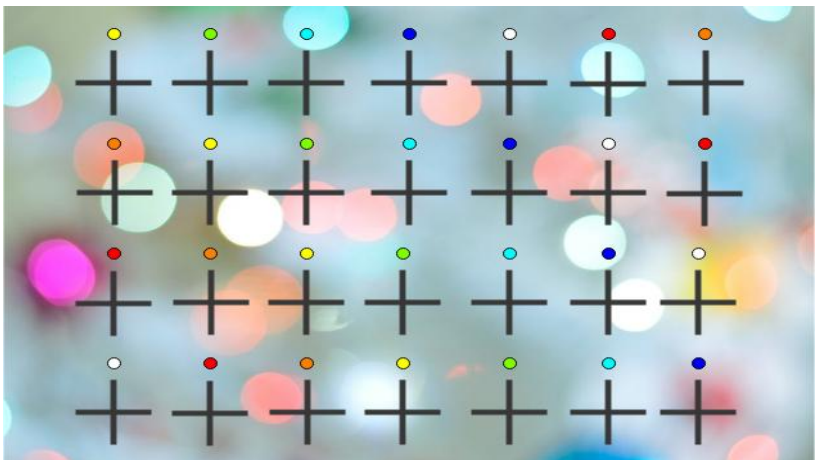

Circles move from right to left horizontally, in a cyclic fashion

Figure 3. Layout of the user interface with moving circles (arrow indicates direction) 


\section{Procedure}

\section{1) Registration Stage}

Initially, a user has to choose a password that consists of a position and a colour for every authentication round. Positions are indicated by the 28 crosshairs on the screen. The user also has to choose among 7 colours (yellow, green, light blue, dark blue, white, red, orange) (See Fig. 2.). The user can choose the number of authentication rounds. The more rounds, the higher the security. Thus the user should choose one crosshair position and one colour for each authentication round.

\section{2) Authentication Stage}

There are two designs for the interfaces for system. The first will be with only stationary colour changing circles (Fig. 2.). During authentication, the circles will be changing colour in a fixed sequence (yellow, green, light blue, dark blue, white, red, orange) on the top of each of the crosshairs. At any one time, each target in the same row will have a different colour circle above it. The mechanism for authentication is as follows: The GUI gives the user a message to get ready to look at the user's password target (crosshair). The user looks at his target and waits for the colours flashing above the target to change to his password colour. At the same time, all the other circles are also flashing and changing colours. The time for the flashing is about 1 second for each colour. Since the colours change in a predictable sequence, the user can anticipate the change to his password colour and uses his peripheral vision of the crosshair adjacent to his target password crosshair to get ready for the change. Once the colour changes to the password colour, user has to press a keyboard button to lock in his choice. This is to avoid the Midas Touch problem. With the click of the button, the fixation time does not have to be lengthy as the button click rather than dwell time locks in the user's choice.

The second design (Fig. 3) will have colour circles which are moving uniformly in one direction. The colour of each circle will not change but instead the circles will move in a cyclic way from right to left. When the circle with the password colour moves into the area encompassed by the crosshair, the user is required to click the button to lock in his choice.

For either of these two designs, the process may be repeated for as many rounds as needed, for increased security.

\section{Password Space}

The password space of this method is calculated as number of targets, $t$ multiplied by number of colours, $c$ and then raised to the power of $n$, which is the number of rounds. The formula is given as:

$$
(t \times c)^{n}
$$

Where

$\mathrm{t}=$ number of targets

$\mathrm{c}=$ number of colours

$\mathrm{n}=$ number of rounds

With 28 targets, 7 colours and 4 rounds, the password space would be $1.476 \times 10^{9}$. In comparison, bank ATM PIN method with 4 digits has a password space of $10^{4}=10000$. Thus the password space is increased.

\section{DISCUSSIONS}

The aim of designed system is to use the ability of humans to see not only in the centre of their focus but also in the peripheral area. Thus it uses a combination of dwell based, peripheral vision and timing based interaction.

A button click together with dwell time avoids the Midas touch problem as well as avoids eye fatigue as user is not forced to fixate on the target for long, only when the button is being pressed. Thus is it expected to be more comfortable to users than purely dwell based methods.

One of the features of this method is that it uses changing colours. This flashing of different colours could result in unease for some users if they are sensitive to it. To accommodate colour blind users, a different choice of colours may have to be made.

The resistance of the designed system against shoulder surfing is expected to be better than that of eye gesture and pursuit based methods as dwell based methods are generally considered to be safer in this respect. It is also expected to be better than purely dwell based methods because it is a combination of dwell and other modalities which increases the password space.

\section{CONCLUSION}

A survey of the related works has been presented. In it we presented the various approaches to eye gaze interaction and summarised the advantages and disadvantages.

Additionally, a method for taking advantage of human field of vision which includes the peripheral area in order to perform authentication was described. The inclusion of this modality could open more possibilities for gaze interaction.

\section{ACKNOWLEDGMENT}

Financial support from the Ministry of Higher Education, Malaysia, under the Fundamental Research Grant Scheme with grant number FRGS/1/2015/SG07/MMU/02/1 is gratefully acknowledged.

\section{REFERENCES}

[1] L. E. Sibert and R. J. K. Jacob. Evaluation of eye gaze interaction. In CHI '00, pages 281-288, 2000. ACM.

[2] A. Bulling, F. Alt, and A. Schmidt. Increasing the security of gaze-based cued-recall graphical passwords using saliency masks. In CHI '12, pages 3011-3020, 2012. ACM.

[3] O. Tuisku, P. Majaranta, P. Isokoski, and K.-J. R“aih“a. Now dasher! dash away!: longitudinal study of fast text entry by eye gaze. In Proc. of the Symposium on Eye tracking Research and Applications, ETRA '08, pages 19-26, 2008. ACM.

[4] D. W. Hansen, H. H. T. Skovsgaard, J. P. Hansen, and E. Møllenbach. Noise tolerant selection by gaze-controlled pan and zoom in $3 \mathrm{~d}$. In Proc. of the Symposium on Eye Tracking Research and Applications ETRA '08, pages 205-212. ACM, 2008.

[5] Vidal, M., Bulling, A., \& Gellersen, H. (2013, September). Pursuits: spontaneous interaction with displays based on smooth pursuit eye movement and moving targets. In Proceedings of the 2013 ACM international joint conference on Pervasive and ubiquitous computing (pp. 439-448). ACM. 
[6] Cymek, D. H., Venjakob, A. C., Ruff, S., Lutz, O. H. M., Hofmann, S. \& Roetting, M. (2014). Entering PIN codes by smooth pursuit eye movements. Journal of Eye Movement Research, 7(4), 1.

[7] Brooks, M., Aragon, C.R. and Komogortsev, O.V., 2013, June. Perceptions of interfaces for eye movement biometrics. In Biometrics (ICB), 2013 International Conference on (pp. 1-8). IEEE.

[8] Maeder, A., Fookes, C. and Sridharan, S., 2004, October. Gaze based user authentication for personal computer applications. In Intelligent Multimedia, Video and Speech Processing, 2004. Proceedings of 2004 International Symposium on (pp. 727-730). IEE.

[9] Weaver, J., Mock, K. and Hoanca, B., 2011, October. Gaze-based password authentication through automatic clustering of gaze points. In Systems, Man, and Cybernetics (SMC), 2011 IEEE International Conference on (pp. 2749-2754). IEEE.

[10] Majaranta P (2012) Communication and text entry by gaze. In: Majaranta $\mathrm{P}$ et al (eds) Gaze interaction and applications of eye tracking: advances in assistive technologies. IGI Global, Hershey, pp 63-77.

[11] Majaranta, P., MacKenzie, I., Aula, A., \& Räihä, K.-J. (2006). Effects of feedback and dwell time on eye typing speed and accuracy. Universal Access in the Information Society, 5(2), 199-208.

[12] Koesling, H., Zöllner, M., Sichelschmidt, L. \& Ritter, H. (2009). With a flick of the eye: Assessing gaze-controlled human-computer interaction. Cognitive Systems Monographs, Human Centered Robot Systems: Cognition, Interaction, Technology (83-92). Berlin: Springer.

[13] Jacob, R. J. (April 1991). The use of eye movements in human-computer interaction techniques: What You Look At is What You Get. ACM Transaction on Information Systems, 9(3), 152-169.

[14] Ohno, T., Mukawa, N., \& Kawato, S. (2003, April). Just blink your eyes: A head-free gaze tracking system. In CHI'03 extended abstracts on Human factors in computing systems (pp. 950-957). ACM.

[15] K. Grauman, M. Betke, J. Lombardi, J. Gips, and G. Bradski, "Communication via Eye Blinks and Eyebrow Raises: Video-Based Human-Computer Interfaces." Universal Access in the Information Society, 2(4), 359-373, November 2003.

[16] Heikkilä, H. \& Räihä, K.-J. (2009). Speed and accuracy of gaze gestures. Journal of Eye Movement Research, 3(2), 1-14.

[17] De Luca, A., Weiss, R., \& Drewes, H. (2007). Evaluation of Eye-Gaze Interaction Methods for Security Enhanced PIN-Entry. Proceedings of the 19th australasian conference on computer-human interaction: entertaining user interfaces (199-202). Adelaide, Australia: ACM.

[18] Drewes, H., \& Schmidt, A. (2007). Interacting with the Computer using Gaze Gestures. INTERACT 2007, 11th IFIP TC 13 Int. Conference, Part II (475-488). Springer.

[19] Møllenbach, E., Hansen, J. P., Lillholm, \& Martin. (2013). Eye Movements in Gaze Interaction. Journal of Eye Movement Research, $6(2), 1-15$

[20] Møllenbach, E., Hansen, J. P., Lillholm, M., \& Gale, A.G. (2009). Single stroke gaze gestures. In Proceedings of the 27th international Conference Extended Abstracts on Human Factors in Computing Systems (CHI '09). ACM, New York, NY, 4555-4560.

[21] Duchowski AT, Vertegaal R (2000) Eye-based interaction in graphical systems: theory and practice. Course 05, SIGGRAPH 2000. Course notes. ACM, New York.

[22] Hillstrom AP, Yantis S (1994) Visual motion and attentional capture. Percept Psychophys 55(4):399-411.

[23] Yvonne Hwei-Syn Kam \& Lip-Yee Por (2016), EyeFlash: Eye Gaze Authentication by Making Use of Peripheral Vision. Short paper presented at the 11th Asia Pacific International Conference on Information Science and Technology (APIC-IST). Unpublished conference paper.

\section{AUTHORS PROFILE}

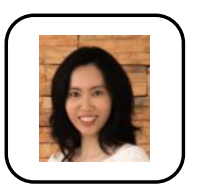

Yvonne Kam has a first class Bachelor of Engineering (Hons) Electronics Majoring in Multimedia degree and a Masters in Engineering Science (MEngSc) in the topic of Image processing, from Multimedia University (MMU). She is a researcher and lecturer in the Faculty of Engineering in MMU and is currently pursuing her $\mathrm{PhD}$ in Computer Science in University of Malaya on the topic of graphical passwords. As part of her $\mathrm{PhD}$, she has developed and implemented applications for security authentication.

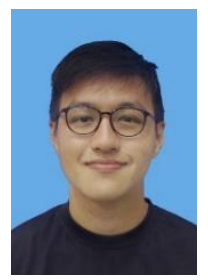

Kar Wing Kwong was a final year engineering student in Multimedia University, Cyberjaya when he contributed to this paper. His final year project was related to this paper, titled as Graphical Login System using Eye-Tracking. Now he holds a Bachelor's of Engineering in Electronics Engineering.

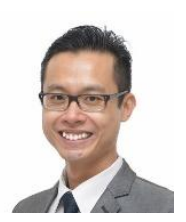

Ir. Dr. Vik Tor Goh received his B.Eng. (Hons.) Electronics and M.Eng.Sc. degrees from Multimedia University, Malaysia. Following that, he obtained a $\mathrm{PhD}$ in Computer Science from Queensland University of Technology, Australia in 2010 for his research in intrusion detection. He was awarded the (ISC)2 Information Security Scholarship in 2007 and 2008 for his pioneering work in intrusion detection. His current research interests include information security, IR4.0, and IoT. In addition to a being a qualified Professional Engineer (PEng, BEM) and Chartered Engineer (CEng, IET), he is also certified as a CISSP. 